\title{
Psychopathology of perpetrators of fabricated or induced illness in children: case series
}

\author{
Christopher Bass and David Jones
}

\section{Background}

Munchausen's syndrome by proxy (recently renamed fabricated or induced illness) is a rare form of child abuse, but relatively little is known about the psychopathology of the perpetrators.
Aims
To examine the medical, psychiatric, social work and forensic records of mothers referred for detailed psychiatric assessment from 1996 to 2009.

\section{Method}
Twenty-eight consecutive individuals with a putative diagnosis of fabricated or induced illness were referred to the authors for detailed psychiatric assessment and recommendations about management (25 from family courts). We scrutinised all medical and psychiatric records and interviewed them, as well as informants.

\section{Results}
In total, 16 (57\%) had evidence of a current somatoform disorder, and factitious disorders (either past or current) were identified in 18 (64\%): 11 participants had both

somatoform and factitious disorders. Nine participants (32\%) had non-epileptic attacks. We found evidence of pathological lying (pseudologia fantastica) in 17 (61\%) of the participants; in some there were key links between early abusive experiences, the development of pathological lying and the eventual fabrication of illness in the child victim.

\section{Conclusions}

A chronic somatoform disorder or factitious disorder (or both) was detected in almost two-thirds of the participants. Over half of the mothers exhibited pathological lying, in some dating from adolescence, and this often continued into adult life eventually involving the child in a web of deceit and abuse. Psychiatrists whose work brings them into contact with women with chronic somatoform or factitious disorders, especially if there is evidence of lying from an early age, should always be alert to the impact of these illnesses on any dependent children.

\section{Declaration of interest}

None.
Fabricated or induced illness (previously called Munchausen's syndrome by proxy) is a form of child abuse in which a parent falsifies illness in a child by fabricating or producing symptoms and presenting the child for medical care while disclaiming knowledge of the cause of the problem. ${ }^{1}$ The disorder has attracted considerable recent interest and controversy. ${ }^{2,3}$ It has even been suggested that the syndrome does not exist, and is itself a 'fabrication' by the medical profession. ${ }^{4}$

We believe that this adverse publicity has drawn attention away from key aspects of induced illness: it is a form of child abuse; it is, in the majority of cases, perpetrated by the mother; its detection and assessment requires detailed and painstaking enquiry involving many different disciplines.

Although there are a number of published articles about the victims of this type of abuse (see Sheridan for a comprehensive review $^{5}$ ) there is a dearth of information about the perpetrators and their backgrounds. In the only systematic study known to us, Bools et $a l^{6}$ reported that three-quarters of women had chronic somatoform disorders and that an even higher proportion had coexisting personality disorders.

Over a period of 15 years we have carried out systematic evaluation of a number of mothers referred (mostly from the courts) with a putative diagnosis of fabricated or induced illness. The family courts in a number of circumstances request detailed medical reports. In most cases this is to establish whether the mother has a treatable mental disorder, and whether reunification of mother and child is possible after an episode of fabricated or induced illness has been demonstrated or is suspected in a child, who may have been taken into care. In these circumstances decisions about the mother's ability to care for the child's safety have to be established. Rarely a report is requested in a woman with a known history of factitious illness who becomes pregnant (these are described elsewhere). One of the advantages of carrying out medico-legal reports for the court is that the decision is made only after review of a considerable amount of not only medical records but also detailed social work and forensic records as well as school records, supplemented of course by an interview with the mother (and where relevant, the father). In this survey we set out to characterise the psychopathology of a consecutive series of people with fabricated or induced illness referred to us since 1996 for detailed psychiatric assessment.

\section{Method}

All but 3 of the 28 participants with a putative diagnosis of fabricated or induced illness were referred from the courts for assessment (the remaining 3 mothers were referred from children's Social Services). Bools et al ${ }^{6}$ noted that 'high levels of psychiatric disturbance, identified in many of the mothers, may be at times very well-concealed from the observer and possibly from the subjects themselves. A detailed medical and psychiatric history and history of antisocial behaviour obtained from reliable sources is necessary to complement the examination of the mental state'. The purpose of the evaluation was to provide information for the court to help in forming legal decisions. Because of this, assessment was painstaking, and guided by the recommendations consistent with those made by Sanders \& Bursch. ${ }^{7}$ For all but 1 of the 28 participants, scrutiny of a complete set of the general practitioner (GP) and medical notes of the mother was supplemented by social work reports and case conferences and, where relevant, written reports from the guardian of the child. 
For two individuals video evidence (usually police interviews with the children) was supplied, and this material was reviewed and summarised before interview with the mother and spouse. In some participants it was possible to discuss the clinical details of the case with the participant's GP. The investigative sequence is shown in Appendix 1.

Biographical data were recorded systematically; in particular, evidence of any physical or childhood sexual abuse or time spent in foster care, and occupational history was noted. Medical records were examined for evidence of any neurological disorders such as epilepsy, head injury, skull fracture or any other medical disorder. In all but one participant full copies of GP notes were available. Details of surgery and medical use were recorded, as was use of prescribed medication. We defined somatisation disorder, using ICD-10 diagnostic criteria for research, as a history of at least 2 years' complaints of multiple and variable physical symptoms that cannot be explained by any detectable physical disorders. Each participant had a total of 6 or more symptoms from a list of 14 symptoms, divided into four groupings (F45.0). ${ }^{8}$ Factitious disorder was defined as intentional production or feigning of symptoms and/or self-infliction of wounds in order to produce symptoms. ${ }^{8}$

Details of any psychiatric history were noted, in particular, admissions to psychiatric hospital and psychiatric diagnoses established in summaries, episodes of self-harm and substance misuse. Specific details of pathological lying (pseudologia fantastica) were recorded and wherever possible the age when this first occurred was noted. Pathological lying was defined as 'falsification entirely disproportionate to any discernible end in view, which may be extensive and very complicated, and may manifest over a period of years or even a lifetime.' The lying or deception in these cases was not confined solely to the fabrication of illness in the child(ren), and usually involved other aspects of the participant's life, for example, fraud and interpersonal relationships. Finally, details of the type of fabricated or induced illness in the children were noted.

\section{Ethical considerations}

The participants were not approached to ask for permission to include them in this paper. This is because it was thought that asking permission in such a sensitive matter might place them under unreasonable duress. We sought ethical guidance from a number of sources. The Medical Defence Union advised that we comply with General Medical Council guidelines to ensure that the data be anonymised so that no case can be identified. We have paid scrupulous attention to this and have anonymised the data presented. We also consulted the local Clinical Ethics Advisory Group and the National Information Governance Board who provided similar advice.

\section{Results}

\section{Biographical data}

All 28 participants were women with a mean age of 31.3 years (range 21-48, s.d. $=7.2), 12(43 \%)$ were married. Only four had been employed in 'healthcare professions', as (auxiliary) nurses. The majority were either unemployed $(n=15)$ or on long-term disability benefit (disability living allowance, $n=7$ ) at the time of the assessments.

\section{Developmental data}

All but four of the participants had experienced a loss or separation from a parent before the age of 11 years. A total of $15(54 \%)$ of the 28 women had been subjected to severe abusive experiences as children and $11(39 \%)$ had spent time in foster care. Three participants had grown up in families where a parent had been absent through imprisonment. Twelve (43\%) reported childhood sexual abuse and seven (25\%) severe physical abuse, which led in two individuals to skull fractures during the first year of life. For some participants these early experiences appear to have had a direct bearing on deceptive behaviour in adult life. From early childhood some participants began to feign symptoms in order to avoid beatings, or to prevent contact visits with abusive parents/carers. The disturbed and disruptive backgrounds led in 17 participants (61\%) to referral to child guidance/adolescent psychiatry clinics (mean age at referral of 14.14 years, s.d. $=1.96$, range 9-16).

\section{Medical histories}

All but 1 of the 28 patients had extensive medical and GP notes, documenting contact with both primary and tertiary care health services. Despite this high rate of attending, there was little evidence of demonstrable physical disease except skull fracture in two, severe congenital dislocation of the hip in one (wheelchair bound), bicornuate uterus in one and chronic endometriosis in another. Three participants had had a hysterectomy and one a reverse sterilisation.

The most common medical diagnoses were epilepsy and 'asthma', and two women had diabetes. Although five participants had received treatment for epilepsy, the diagnoses were equivocal in three. Of the remainder, one had childhood epilepsy and another photosensitive seizures. Nine participants (32\%) had evidence of pseudoseizures (psychogenic non-epileptic seizures) and nine had been diagnosed with asthma (four had both). Of the nine who had been diagnosed with asthma, six were receiving treatment for it, although the diagnosis was equivocal in all of them (not confirmed by lung function tests). Factitious asthma was established in one of these. Significantly, all six of the women in receipt of treatment for asthma also reported pseudoseizures (see below).

\section{Medication use}

Six of the nine participants diagnosed with asthma were in receipt of inhalers and one with factitious asthma was prescribed prednisolone. Three were receiving anticonvulsant medication, a further three opiates for pain and one a diuretic for unspecified reasons. The two with diabetes were prescribed metformin and insulin respectively. Only three were taking antidepressants at the time of the assessment.

\section{Psychosocial and forensic data}

Ten participants had forensic histories: six had convictions for shoplifting, two for arson and another two for police 'harassment' following a barrage of hoax telephone calls. Of the 17 (61\%) who had been referred to child and adolescent psychiatry services, the most common reasons cited were 'disruptive behaviour': other reasons included repeated self-harm; anxiety and depression; school refusal; eating disorders; encopresis and dealing with the consequences of childhood sexual abuse.

As adults, the majority had had contact with psychiatric services. In total, $6(21 \%)$ had been admitted to psychiatric hospitals (3 the subject of the Mental Health Act) and 20 (71\%) had received out-patient treatment. Fifteen (54\%) had a history of self-harm, with repeated cutting in three. In many cases, however, participants had failed to satisfactorily engage with psychiatric services or had defaulted from follow-up. Half had received psychiatric treatment for a mood disorder, but by far the most common diagnosis documented in the psychiatric 
records was a personality disorder $(n=21,75 \%)$, the most frequent being combinations of antisocial, borderline, histrionic and anxious/dependent.

Scrutiny of the primary care and medical records revealed that 16 participants (57\%) reported persistent and enduring physical complaints for at least 2 years for which no organic cause could be established. In all but 3 of these 16 individuals the physical symptoms had been chronic and lasted for over 5 years, with a mean duration of 16.1 years (s.d. $=6.1$, range $7-27$ ). They were particularly high users of neurology, gastroenterology, obstetric and gynaecology services, as well as frequent attendees in the accident and emergency department. In diagnostic terms these individuals satisfied diagnostic criteria for somatisation disorder. ${ }^{8}$ These women reported symptoms affecting many different organs, with abdominal pain, breathlessness and nonepileptic seizures being the most common. In 18 (64\%) there was evidence of fabricated or factitious illness (coexisting with somatisation disorder in 11) but occurring 'in isolation' in 4 of them. Eight women had coexisting somatoform and factitious disorders with histories of self-harm (Fig. 1). In five participants $(19 \%)$ there was evidence of at least one false pregnancy (pseudocyesis).

Fabricated illnesses in the women included epilepsy, diabetes and asthma, and although the diagnosis of factitious disorder had been suspected in some (who not infrequently received a discharge diagnosis of Munchausen's syndrome), none of these individuals had been confronted about their deceptions by their medical attendants.

\section{Pathological lying or pseudologia fantastica}

In 17 participants $(61 \%)$ lying and fabricating stories had been documented in and by a variety of different sources. These included social work and psychiatric records, the minutes of child protection conferences, medical and psychiatric notes, foster care records, police records and by members of the family. Occasionally, school records contained evidence of lying and deception. The earliest documented record of lying was at age 6, and in others it was often documented during adolescence.

In most cases the lying was established early in life and persisted through adolescence and adult life as an enduring personality trait or characteristic. In some it appeared to be related to stressful life events, but it was associated with episodes of pathological wandering in only one. In others this took the form of repeated telephone hoax calls, which was sometimes associated with escalation of treatment-seeking behaviour, lying and induced illness in the child.

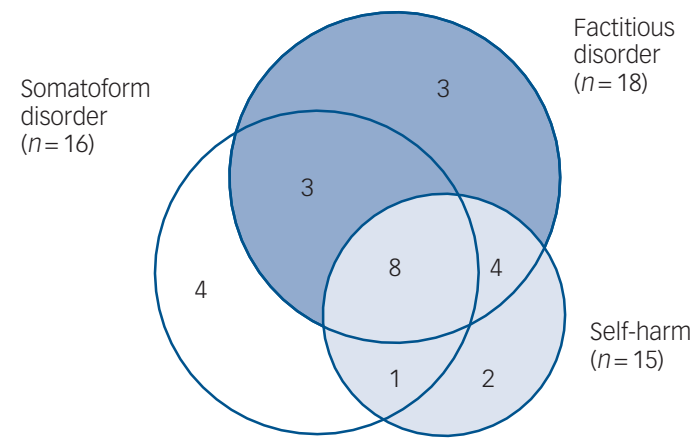

Fig. 1 Association between factitious disorder, somatoform disorder and self-harm in the 28 participants.

\section{The children: victims of the abuse}

A total of $21(75 \%)$ of the 28 children were under 5 years old at the time of the referral, and 18 (64\%) were female. There were two pairs of twins, and both of one pair was abused. Twenty-one children had siblings, and in general only one child in a sibship was the victim of abuse. In 20 there was 'fabrication' of illness (for example the mother telling a school nurse that the child was dying of cancer or had epilepsy) and in 13 poisoning or induction of illness. Seven $(23 \%)$ of the mothers had carried out both induction and fabrication.

Seventeen children (61\%) had received diagnoses of 'neurological' diagnoses (most commonly epilepsy, but also anoxic episodes, ataxia or attention-deficit hyperactivity disorder). Examination of their mothers' records revealed that two had received a diagnosis of epilepsy and seven had psychogenic nonepileptic seizures. This coexistence of pseudoneurological symptoms in the mothers and their children will be discussed below. Two of the children were in wheelchairs.

We attempted to determine how these cases of fabricated illness in the children were detected, i.e. who first raised the alarm. For seven it was Social Services; five each from paediatric services, the child's school, and adult mental health services; two from family members; and one each from child psychiatry services, health visitors, GP and self-referral.

\section{Discussion}

\section{Main findings}

We found high rates of chronic somatoform disorders in these women, with enduring somatoform disorders in 57\% and fabricated symptoms in 64\% (11 individuals had both chronic somatisation and factitious illness). In many participants the use of healthcare services was chaotic, with frequent visits to different accident and emergency departments, frequent changes of GP (often instigated by the GP) and lack of continuity of care. Discussion with the individual's GP seldom yielded any useful information because the GP's knowledge of the individual was often fragmented and brief. This association between persistent somatisation and factitious illness is known to occur in a subset of very high users of medical and surgical services. ${ }^{10}$ We were able to establish a positive diagnosis of factitious disorder in our participants because we had access to so much clinical data from different sources.

Of the medically unexplained symptoms, pseudoneurological complaints (faints and pseudoseizures) and gastroenterological symptoms (abdominal pain, nausea) were the most common. Nine people (32\%) reported pseudoseizures and, significantly, epilepsy and anoxic episodes had been diagnosed in seven of their children. Of the nine women who had been diagnosed as asthmatic, four also reported pseudoseizures. The association between pseudoseizures and reported asthma has been noted by others, ${ }^{11}$ and it has been suggested that anxiety, hyperventilation and dissociative elaboration might account for the observed association. Both asthma and anxiety/hyperventilation may be important risk factors for the development of pseudoseizures, but the reported asthma itself may be psychogenic in origin, as seems likely in the majority of our participants. The finding of pseudocyesis or false pregnancy in a fifth of mothers is of interest; to our knowledge it has not been reported before in these women.

Over half our participants had histories of self-harm, which often began in adolescence and in some continued into adult life. Only three had either current or lifetime histories of substance misuse, and ten had forensic histories; most often this involved shoplifting, theft or arson. Two women received custodial 
sentences after the assessment. Although we did not formally measure personality disturbance or disorder in our participants, a diagnosis of personality disorder had been established in the psychiatric notes in about three-quarters of those individuals who had received psychiatric treatment. Often coexisting with the personality difficulty was a defensive or sometimes frankly hostile attitude towards Social Services, especially among those women who had reported negative experiences while in care.

\section{Methodological issues and limitations}

These women represent a consecutive case series referred to one unit over a period of 15 years and so it is possible that they were not representative of individuals with fabricated or induced illness. All but three had been referred from the family courts for psychiatric assessment after episodes of alleged fabricated or induced illness, accompanied by a request for an opinion on the suitability of reunification of mother and child. Our sample is therefore biased towards those women who are detected, suspected and referred for detailed assessment, rather than a description of all women who carry out fabricated or induced illness behaviour. These limitations aside, however, we were able, because of the stringent requirements of the court, to obtain detailed medical and social histories for all of our participants except one. This included all relevant primary care data, which were invaluable in establishing the history and duration of each person's physical and psychological symptoms. The similarities between our data and the only other major published series ${ }^{6}$ lead us to believe that our sample is not atypical or unrepresentative. Furthermore, just over half the children in our series were victims of fabricated epilepsy or anoxic episodes, which is similar to the $42 \%$ reported in a well-known North American review. ${ }^{12}$ Seizures have also been reported to be the most common presentation of fabricated illness in children. ${ }^{13}$ It remains possible that there is a wider group of people who carry out fabricated or induced illness behaviours, perhaps a milder version, who do not necessarily respond with hostility and lies when detected.

\section{Early childhood experiences and intergenerational factors}

The most striking abnormality in the childhood experiences of these women was the high rate of early family disruption and loss. Only 2 of the 28 had not experienced loss of a parent (through death, separation/divorce or removal of a parent to prison) before the age of 11 years, and over a third had spent time in foster care during their formative years. High rates of physical or sexual abuse have been reported by others, ${ }^{14}$ and in another study insecure attachment as well as high rates of unresolved trauma and loss reactions was identified in these mothers. ${ }^{15}$

The disruptive and turbulent childhoods experienced by the majority of these women is reflected in the very high referral rates to child and adolescent psychiatry services. Over half of the sample was assessed in these services, and three-quarters went on to have contact with adult psychiatric services. It is also worth noting that studies of children and adolescents with factitious disorder referred to child and adolescent consultation liaison services have revealed strikingly similar biographical and clinical characteristics to our sample. ${ }^{16}$

Recent studies have found support for the hypothesis that the children of parents with somatoform disorders are at increased risk of having high rates of contact with medical services as well as developing abnormal health beliefs. ${ }^{17,18}$ Our findings confirm this intergenerational component, and also suggest an important association between individuals who fabricate symptoms first in themselves and later in their children. ${ }^{19}$ For this reason our findings emphasise the important implication of establishing a diagnosis of factitious disorder in women of childbearing age. Because of the increased subsequent risk of fabricated illness occurring in a child, any pregnant woman who has received this diagnosis should undergo a comprehensive multidisciplinary assessment to assess the risk of harm to the unborn child.

\section{Importance of pathological lying}

Examination of social work, medical and psychiatric notes revealed that over half of the participants exhibited pathological lying (sometimes referred to as pseudologia fantastica ${ }^{20}$ ) and in some this dated from a very early age; 6 years was the earliest recorded. These lies were often compulsive, habitual and sometimes self-aggrandising, and occurred throughout adolescence in many. In some they emerged at times of life stress and the episode of fabricated or induced illness was often accompanied by a re-emergence of lying and other deceitful activity such as repeated hoax telephone calls to police about 'harassment', which occurred in two women, financial fraud in one and nomadic wandering in another.

Enquiry into the motivations for feigning symptoms revealed links with these early abusive or key formative experiences in only about a third of the 28 women. For example, some patients 'made up' symptoms in order to avoid beatings or to prevent contact visits with abusive parents and others reported that they felt more cared for in hospital. In these cases developmental links could be detected between early disruptive and abusive experiences and later patterns of deceptive illness behaviour. The role of pathological lying in this behaviour is mentioned above.

We have already mentioned the possible links between early adverse experiences and subsequent illness behaviour: in over half our sample pathological lying was a key component of the psychopathology. It was often commented on in social work notes or case conferences. When confronted about the lying, individuals often responded by denial or disavowal of their contribution to the deception. As expected, all but 2 of the 17 participants exhibiting pathological lying also reported factitious physical symptoms in their own right.

\section{Implications for clinical practice}

Factitious disorder in adults and fabricated or induced illness (in children) can co-occur, so the detection of one should trigger a search for the other. Psychiatrists whose clinical work brings them into contact with parents with chronic somatoform and factitious illnesses should be alert to the impact of these disorders on the individuals' children. Our retrospective cohort does not allow us to identify which women with somatisation disorder and/or factitious disorder are at risk of carrying out this form of abuse. It may be that some women with a somatising disorder do not present a risk to their child, perhaps those at the milder end of the spectrum and those who respond to early identification without lying, hostility or deceit. Our sample necessarily describes a group who were detected, suspected and then referred, often from the family courts. It is probable therefore that our sample describes a severe end of a broader spectrum, although longitudinal studies of child-rearing women with a somatising disorder would be required to answer this question. At this point we are unable to speculate as to what proportion of women with a somatising disorder would go on to present a risk to their child. However, some 'risk factors' can be identified from our data, and identification of these should alert clinicians to those women 
who may be at increased risk of inducing abnormal illness behaviour in their children. These risk factors are shown in Appendix 2.

Our findings also suggest that no clinician should attempt to carry out an assessment of a woman suspected of this form of child abuse without access to extensive previous medical (and other) records. Neither should any opinion be expressed by the examining doctor about the mother's competence to look after a child based on a single assessment in an out-patient clinic. Our findings also imply that great care be taken in validating symptom reports by these women, especially those where evidence of pathological lying is suspected or has been established. In practice this means that the clinician needs to corroborate clinical data using a variety of different sources, both medical and nonmedical, and a degree of healthy scepticism is required throughout the management of such cases.

Our sample of women had experienced high rates of early adverse experiences, including neglect and abuse as children and high rates of foster care. In adult life, somatoform disorders and factitious disorders also coexisted in nearly two-thirds of the women, with evidence of self-harm in about a half. The most frequently reported physical complaints were non-epileptic seizures and 'psychogenic' asthma, as well as gastrointestinal symptoms. Significantly, there were often similarities between the 'functional' symptoms reported by the mothers and the pseudoneurological symptoms fabricated or induced in their children. Such individuals are notoriously difficult to manage, but it is clear that the challenge of pregnancy and childbirth provide additional stressors for these women that invariably lead to ambivalent or hostile impulses towards their children. ${ }^{21}$

Three-fifths of the mothers exhibited pathological lying from early adolescence and often this continued into adult life. When these mothers became distressed their lying invariably eventually involved the children, who then became the victims of fabricated and induced illness. Pathological lying is a rare but important symptom, and can occur in a variety of different clinical settings. ${ }^{9}$ Our findings suggest that detection of pathological lying in adolescent or young women should alert medical and social work staff to its potential significance when these women become pregnant. In some cases the pathological lying became established in early life to avoid some feared consequence or to elicit a desired response from others. It is important to stress, however, that the motivation for the induced illness in children was unclear in two-thirds of individuals. Reunification of the child with the mother can be attempted, but only in very carefully selected individuals. ${ }^{22}$

Christopher Bass, MA, MD, FRCPsych, Department of Psychological Medicine, John Radcliffe Hospital, Oxford; David Jones, FRCPsych, Department of Psychiatry, Warneford Hospital, Oxford, UK

Correspondence: Christopher Bass, MA, MD, FRCPsych, Department of Psychological Medicine, John Radcliffe Hospital, oxford OX3 9DU. Email: christopher.bass@oxfordhealth.nhs.uk

First received 25 Nov 2009, final revision 28 Feb 2011, accepted 2 Mar 2011

\section{Appendix 1}

\section{Assessment of the alleged perpetrator: preparation}

(a) Medical and nursing records of the child's mother: (i) hospital

(ii) primary care (handwritten and typed).

(b) Medical and nursing records of all involved children.

(c) Social work records/reports/case conferences. (d) Police records, videos

(e) Legal documents:

(i) statement of mother and father

(ii) report of child's guardian.

(f) Interview with mother and father.

(g) Interview grandparents.

(h) Telephone interview with GP, social workers, paediatrician and guardian.

(i) Multidisciplinary case conference (ideal).

\section{Appendix 2}

\section{Risk factors identified in our sample of mothers for creating abnormal illness behaviour in children}

Remote risk
(a) Loss or separation from parent.
(b) Abuse/neglect.
(c) Foster care.
(d) History of lying in adolescence.
(e) History of self-harm.

\section{Recent risk}

(a) Current somatoform disorder.

(b) Current factitious disorder.

(c) In receipt of disability living allowance.

(d) Child missing school.

(e) Frequent visits to doctors (symptoms unexplained).

AND . . .

(a) Frequent moves of house (and GP).

(b) Parent requests disability living allowance for child

\section{References}

1 Department of Health. Safeguarding Children in whom Illness is Fabricated or Induced. Department of Health, 2003 (http://www.dh.gov.uk/en/ Publicationsandstatistics/Publications/PublicationsPolicyAndGuidance/ DH_4008714).

2 Bools C. Fabricated or Induced IIIness in a Child by a Carer. A Reader Radcliffe Publishing, 2007.

3 Anon. Fabricated or induced illness by carers: a complex conundrum. Lancet 2010; 375: 433 .

4 Persaud R. Keeping mum over child abuse. BMJ 2005; 330: 152.

5 Sheridan M. The deceit continues: an updated literature review of Munchausen syndrome by proxy. Child Abuse Negl 2003; 27: 431-51.

6 Bools CN, Neale B, Meadow SR. Munchausen syndrome by proxy: a study of psychopathology. Child Abuse Negl 1994; 18: 773-8.

7 Sanders M, Bursch B. Forensic assessment of illness falsification, Munchausen by proxy, and factitious disorder, NOS. Child Maltreat 2002; 7: $112-24$.

8 World Health Organization. The ICD-10 Classification of Mental and Behavioural Disorders: Diagnostic Criteria for Research. WHO, 1993.

9 Dike C, Baranoski M, Griffith EE. Pathological lying revisited. I Am Acad Psychiatry Law 2005; 33: 342-9.

10 Fink P. Surgery and medical treatment in persistent somatising patients. J Psychosom Res 1992; 36: 439-47.

11 de Wet C, Mellers J, Gardner W, Toone B. Pseudoseizures and asthma. J Neurol Neurosurg Psychiatry 2003; 74: 639-41.

12 Rosenberg D. Web of deceit; a literature review of Munchausen syndrome by proxy. Child Abuse Neglect 1987; 11: 547-63.

13 Barber M, Davis P. Fits, faints, or fatal fantasy? Fabricated seizures and child abuse. Arch Dis Child 2002; 86: 230-3. 
14 Gray J, Bentovim A. Illness induction syndrome: paper I - a series of 41 children from 37 families identified at the Great Ormond Street NHS Trust. Child Abuse Neglect 1996; 20: 655-73.

15 Adshead G, Bluglass K. Attachment representations in mothers with abnormal illness behaviour by proxy. Br J Psychiatry 2005; 187: 328-33.

16 Ehrlich S, Pfeiffer E, Salbach H, Lenz K, Lehmkuhl U. Factitious disorder in children and adolescents: a retrospective study. Psychosomatics 2008; 49: 392-8.

17 Craig $\mathrm{TK}$, Cox AD, Klein $\mathrm{K}$. Intergenerational transmission of somatization behaviour: a study of chronic somatisers and their families. Psychol Med 2002; 32: 805-16.
18 Marshall T, Jones DPH, Ramchandani PG, Stein A, Bass C. Intergenerational transmission of health beliefs in somatoform disorders. Exploratory study Br J Psychiatry 2007; 191: 449-50.

19 Feldman $\mathrm{M}$, Rosenquist $\mathrm{P}$, Bond J. Concurrent factitious disorder and factitious disorder by proxy. Double jeopardy. Gen Hosp Psychiatry 1997; 19 24-8.

20 King B, Ford C. Pseudologia fantastica. Acta Psychiatr Scand 1988; 77: 1-6.

21 Adshead G, Bluglass $K$. A vicious circle: transgenerational attachment representations in a case of factitious illness by proxy. Attach Hum Dev 2001; 3: 77-95.

22 Berg B, Jones D. Outcome of psychiatric intervention in factitious illness by proxy (Munchausen's syndrome by proxy). Arch Dis Child 1999; 81: 465-72.

\section{extra}

\section{4:48 Psychosis (excerpt)}

\section{Sarah Kane}

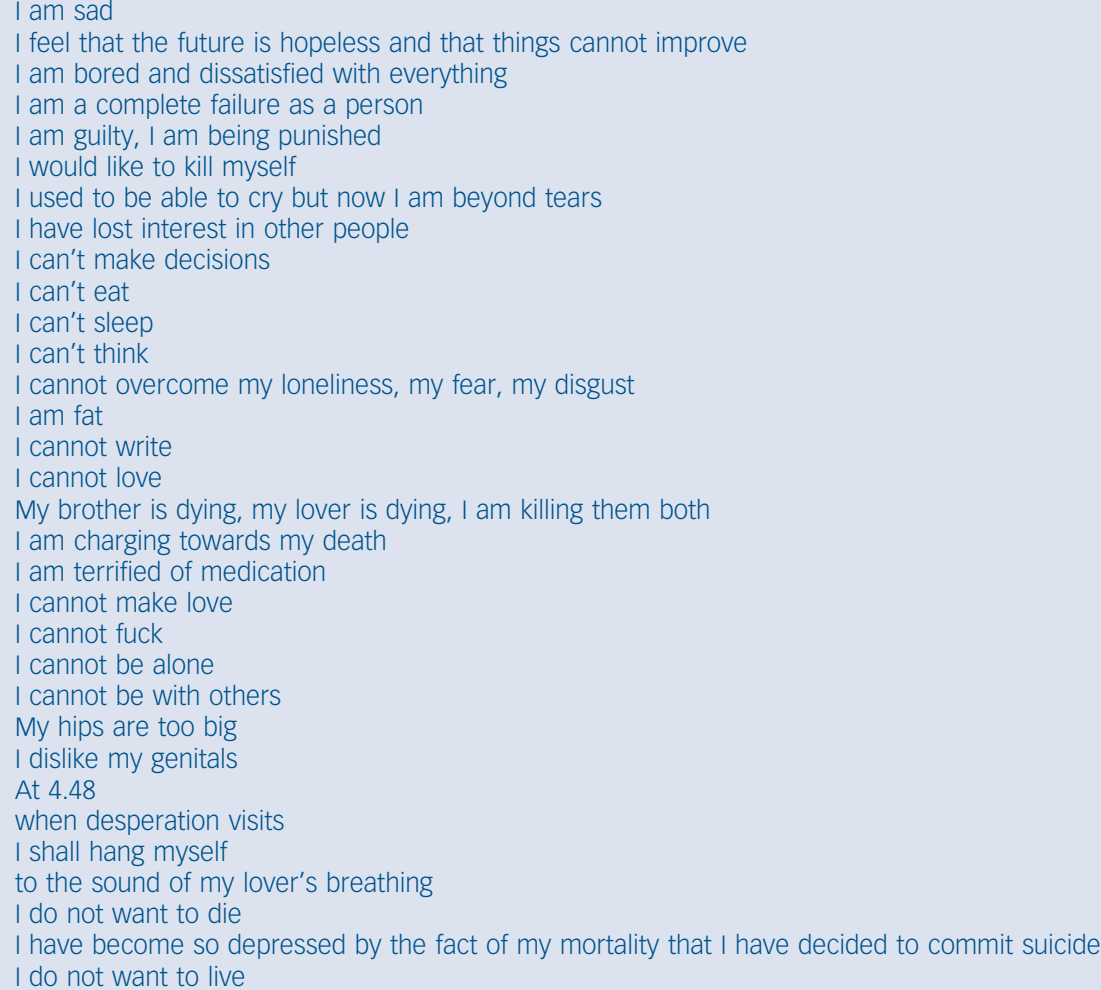

